\title{
Cistatina C: Um Marcador de Função Renal Promissor em Doentes com Lúpus Eritematoso Sistémico?
}

\author{
Cystatin C: A Promising Marker of Renal Function in Patients with Systemic \\ Lupus Erythematosus?
}

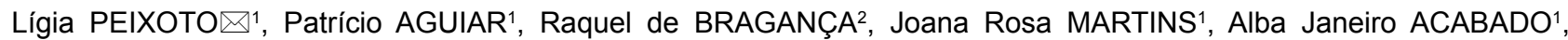
José Luís DUCLA-SOARES ${ }^{1,2}$

Acta Med Port 2015 May-Jun;28(3):333-341

\section{RESUMO}

Introdução: A cistatina C possui uma correlação superior com a taxa de filtrado glomerular e um prognóstico clínico mais significativo do que a creatinina. Procurou-se averiguar se constitui um marcador de função renal diferente da creatinina (cistatina $C$ potencialmente superior à creatinina), em doentes com lúpus eritematoso sistémico.

Material e Métodos: Foram avaliados 37 doentes com lúpus eritematoso sistémico, sem evidência de nefrite lúpica activa. Determinouse a cistatina $\mathrm{C}$ sérica por nefelometria e a creatinina pelo método de Jaffe modificado. Compararam-se cinco fórmulas: Chronic Kidney Disease - Epidemiology Collaboration cystatin; Chronic Kidney Disease - Epidemiology Collaboration creatinine-cystatin; CockcroftGault, Modification of Diet in Renal Disease e Chronic Kidney Disease - Epidemiology creatinine, utilizando-se esta última como referência. Analisou-se a influência de factores clínicos e laboratoriais na variação da cistatina C, por regressão linear multivariada.

Resultados: A cistatina $C$ encontrava-se isoladamente elevada em dez participantes, ao invés de nenhuma elevação isolada da creatinina, sendo esta diferença significativa $(p=0,002)$. Verificou-se uma diferença entre a taxa de filtrado glomerular estimada pela Chronic Kidney Disease - Epidemiology Collaboration cystatin e pela Chronic Kidney Disease - Epidemiology Collaboration creatinine $\left(-6,0541 \mathrm{~mL} / \mathrm{min} / 1,73 \mathrm{~m}^{2}, p=0,07\right)$, mais acentuada para taxas de filtração glomerular mais baixas. Assim, a fórmula Chronic Kidney Disease - Epidemiology Collaboration cystatin reclassificou 4 doentes como tendo doença renal crónica de novo e um doente como não tendo doença renal crónica $(p=0,375)$. A cistatina $C$ foi influenciada significativamente apenas pela idade $(p<0,001)$.

Discussão: Vários estudos demonstraram que a cistatina $C$ melhora a definição de doença renal crónica, permitindo uma classificação e uma estratificação do risco mais exactas, comparativamente à creatinina. A cistatina $C$ revelou-se, neste estudo, um marcador de função renal promissor nos doentes com lupus, principalmente para taxas de filtrado glomerular mais baixas. A correlação da cistatina $\mathrm{C}$ com a idade para ser um factor confundente, na medida em que existe um declínio fisiológico da taxa de filtração glomerular com o envelhecimento.

Conclusão: A cistatina $\mathrm{C}$ foi potencialmente superior à creatinina e nesta amostra a cistatina $\mathrm{C}$ pareceu detectar mais precocemente do que a creatinina alterações na taxa de filtrado glomerular, podendo ser um melhor método de rastreio de doença renal crónica no lúpus eritematoso sistémico.

Palavras-chave: Cistatina C; Insuficiência Renal; Lúpus Eritematoso Sistémico; Marcadores Biológicos.

\section{ABSTRACT}

Purpose: Cystatin $\mathrm{C}$ has a higher correlation with glomerular filtration rate and a more significant clinical prognosis than creatinine. We sought to determine whether it is a marker of renal function different from creatinine (cystatin C potentially superior to creatinine), in patients with systemic lupus erythematosus.

Material and Methods: 37 patients with systemic lupus erythematosus were evaluated. Serum cystatin C was determined by nephelometry and creatinine by modified Jaffe method. We compared five formulas: Chronic Kidney Disease - Epidemiology Collaboration cystiatin; Chronic Kidney Disease - Epidemiology Collaboration creatinine-cystatin; Cockcroft-Gault; Modification of Diet in Renal Disease and Chronic Kidney Disease - Epidemiology Collaboration creatinine, using the latter as a reference. We analyzed the influence of clinical and laboratory factors in cystatin $C$ variation, using multivariate linear regression.

Results: Cystatin $C$ was singly elevated in ten participants, versus none isolated creatinine elevation, and this difference was significant $(p=0.002)$. There was a difference between the estimated glomerular filtration rate by Chronic Kidney Disease - Epidemiology Collaboration cystatin and by Chronic Kidney Disease - Epidemiology Collaboration creatinine $\left(-6.0541 \mathrm{~mL} / \mathrm{min} / 1.73 \mathrm{~m}{ }^{2}, p=0.07\right)$, more pronounced for lower glomerular filtration rate. Consequently, Chronic Kidney Disease - Epidemiology Collaboration cystatin reclassified 4 patients as having chronic kidney disease de novo and 1 patient as not having chronic kidney disease $(p=0.375)$. Cystatin $\mathrm{C}$ was only significantly influenced by age $(p<0.001)$.

Discussion: Several reports showed cystatin $\mathrm{C}$ as a better marker to define chronic kidney disease, allowing more accurate classification and risk stratification, compared with creatinine. In this study, Cystatin $C$ revealed as a promisor marker of renal function in patient with lupus, mainly in patients with lower glomerular filtration rates. The correlation between age and cystatin $\mathrm{C}$ seems to be a confounding factor, as glomerular filtration rate physiologically declines with ageing.

Conclusion: Cystatin $\mathrm{C}$ was potentially superior to creatinine and in this study and cystatin $\mathrm{C}$ seems to detect changes in glomerular filtration rate earlier than creatinine and may be a better screening method for chronic kidney disease in systemic lupus erythematosus. Keywords: Biological Markers; Cystatin C; Lupus Erythematosus, Renal Insufficiency; Systemic.

\footnotetext{
1. Serviço de Medicina I. Centro Hospitalar Lisboa Norte. Lisboa. Portugal.

2. Faculdade de Medicina. Universidade de Lisboa. Lisboa. Portugal.

$\bowtie$ Autor correspondente: Lígia Peixoto. ligia_peixoto@yahoo.com.br

Recebido: 26 de Agosto de 2014 - Aceite: 21 de Abril de 2015 | Copyright @ Ordem dos Médicos 2015
} 


\section{INTRODUÇÃO}

A doença renal crónica (DRC) constitui um problema de saúde major emergente em todo o mundo, quer pelo aumento da sua incidência e prevalência nas últimas décadas, quer pelo impacto clínico e económico associado, constituindo um factor de risco independente para doença cardiovascular e lesão renal aguda. ${ }^{1}$

Desta forma, o seu diagnóstico precoce assume uma importância inquestionável, para que se possa retardar a progressão da doença e suas complicações. De acordo com a Kidney Disease: Improving Global Outcomes (KDIGO), a DRC pode ser definida como anormalidades na estrutura ou na função renal, presentes por mais de três meses, com implicações para a saúde. ${ }^{2}$ Contempla-se, assim, um período de diminuição funcional, considerando-se a taxa de filtração glomerular (TFG) o melhor indicador da função renal global.

Assim, e sendo a DRC muitas vezes assintomática até estádios mais avançados, o seu diagnóstico e classificação dependem vastamente da utilização de biomarcadores de função renal, área que tem sido amplamente investigada e discutida no panorama científico. ${ }^{3,4}$

A TFG pode ser medida através da clearance de um marcador de filtração exógeno, sendo o gold standard a medição da clearance urinária da inulina durante uma infusão intravenosa contínua. Outros marcadores exógenos possíveis são o iotalamato, o iohexol ou o ácido etilenodiamino tetra-acético (EDTA). ${ }^{5}$ Contudo, a medição da TFG é dispendiosa, invasiva, pouco prática e não está disponível rotineiramente.

Deste modo, a TFG é geralmente estimada (TFGe) a partir da concentração sérica de marcadores endógenos. $\mathrm{O}$ biomarcador ideal seria aquele que fosse produzido a uma taxa constante e fosse eliminado exclusivamente por filtração glomerular. Nestas condições, a sua concentração sérica no estado estacionário reflectiria a TFG.

A creatinina, o marcador clássico da TFG, é um aminoácido que deriva da degradação não enzimática da creatina no músculo. Constitui um bom marcador de função renal pois não se liga a proteínas e não é metabolizada no rim, sendo filtrada livremente no glomérulo. ${ }^{3}$

Contudo, possui reconhecidas limitações, já que a sua concentração sérica não depende exclusivamente da TFG, mas é influenciada largamente por outros factores fisiológicos, sendo a taxa de produção da creatinina proporcional à massa muscular. A sua excreção não depende exclusivamente da filtração glomerular, ocorrendo secreção tubular proximal (10 - 20\%), bem como eliminação extra-renal pelas bactérias da flora gastrointestinal, sendo que estes mecanismos se tornam mais importantes com o declínio da função renal, permitindo manter a concentração sérica da creatinina dentro de valores normais até uma diminuição muito significativa da TFG (cerca de $60 \mathrm{~mL} / \mathrm{min} / 1,73 \mathrm{~m}^{2}$ ) faixa cega da creatinina. ${ }^{4,6}$

Procurou-se ultrapassar a variabilidade inter-individual da produção da creatinina através do desenvolvimento de equações que melhoram o cálculo da TFG, através da in- clusão diversos factores antropométricos, das quais são exemplo a fórmula de Cockroft-Gault, a do estudo Modification of Diet in Renal Disease (MDRD) e a do Chronic Kidney Disease Epidemiology Collaboration (CDK-EPI).3,6,7 Segundo a KDIGO (2012), a equação recomendada para calcular a TFG é a equação da creatinina do estudo CKD-EPI 2009 (CKD-EPI creat), pois demonstrou ser mais exacta e precisa e ter menos viés comparativamente à equação do estudo MDRD, especialmente para TFG mais elevadas. ${ }^{2,8}$

Como marcador alternativo de TFG tem sido investigada a cistatina $C$, uma proteína não glicosilada de baixo peso molecular, pertencente à família dos inibidores das cisteinoproteases, produzida, a ritmo constante, por todas as células nucleadas, livremente filtrada nos glomérulos renais, e por fim reabsorvida e metabolizada nos túbulos proximais. Não existindo secreção tubular ou eliminação extra-renal significativa, a sua concentração periférica depende exclusivamente do ritmo de filtração glomerular, o que a torna promissora como marcador de função renal. A sua produção não depende da massa muscular ou da dieta, revelando-se mais uniforme. Assim, os níveis séricos de cistatina $C$ têm demonstrado consistentemente ter uma correlação superior com a TFG do que a creatinina. , $^{9,10}$

A cistatina $C$, tal como a creatinina, deve ser utilizada para avaliar a função renal a partir de equações que estimem a TFG, sendo a recomendada pela KDIGO a equação CKD-EPI de 2012 (CKD-EPI ${ }_{\text {cist }}$ ).

Provavelmente existirão outros factores independentes da TFG que alteram a concentração da cistatina $\mathrm{C}$ e que não foram contemplados nestas equações. De facto, a produção deste biomarcador parece estar aumentada em situações de turnover celular aumentado, como hipertiroidismo ou doenças malignas, e, apesar da literatura não ser consensual e de serem necessários mais estudos que demonstrem uma correlação causal, coloca-se a hipótese de que estados inflamatórios, diabetes, obesidade, tabagismo ou o uso de corticoesteróides sejam também factores influenciadores. . $^{6,11}$

A KDIGO inclui nas suas mais recentes recomendações para a DRC (2012) várias sugestões relacionadas com a cistatina C.

Uma população a considerar seria a do lúpus eritematoso sistémico (LES). A lesão renal constitui um factor importante de morbilidade e mortalidade nestes doentes, sendo que pelo menos $60 \%$ manifestará algum grau de envolvimento renal durante o curso da doença. ${ }^{12}$ Sendo a nefrite assintomática na maioria dos doentes, a determinação da função renal tem um papel ainda mais relevante no que toca à identificação clínica precoce, estratificação do risco e ajuste das doses terapêuticas, nesta população. ${ }^{13}$

As Recomendações Europeias de 2009 de glomerulonefrite lúpica, sugerem a avaliação da função renal em doentes com LES através dos níveis de creatinina sérica ou pelas diversas equações que estimam a TFG, excepto nos casos em que a TFGe é superior a $60 \mathrm{~mL} / \mathrm{min} / 1,73 \mathrm{~m}^{2}$, situação em que o método aconselhado é a determinação da 
clearance da creatinina (CIC) através da colecção da urina de 24 horas. ${ }^{14,15}$ Contudo, actualmente as recomendações globais de avaliação da função renal sugerem a utilização de equações para estimar a TFG, em detrimento da CIC, método pouco prático, demorado, com elevadas taxas de colecções inadequadas e que tende a sobrestimar a TFG.

A fórmula que melhor se aplica a doentes com LES para estimar a função renal não foi ainda devidamente apurada. Com este estudo pretende-se averiguar se a cistatina $\mathrm{C}$ poderá constituir um marcador de função renal alternativo e potencialmente superior à creatinina, em doentes com LES.

Concretamente, pretende-se comparar o viés, a exactidão e a precisão das equações de TFG derivadas da cistatina C (CKD-EPI cist $_{\text {e CKD-EPI }}$ creat-cist $)$, com outros métodos utilizados na prática clínica, derivados apenas da creatinina, nomeadamente, com as fórmulas Cockroft-Gault, MDRD e CKD-EPI ${ }_{\text {creat }}$.

Procura-se ainda verificar se a classificação de DRC (TFGe $<60 \mathrm{ml} / \mathrm{min} / 1,73 \mathrm{~m}^{2}$ ) dos diversos doentes difere com a utilização dos vários métodos e o de referência.

Por fim, pretende-se determinar se existem factores extra-renais que influenciem os níveis séricos da cistatina $C$.

\section{MATERIAL E MÉTODOS}

Trata-se de um estudo observacional, transversal e prospectivo, no qual foram incluídos doentes com LES seguidos na consulta de Doenças Autoimunes do Serviço de Medicina I do Centro Hospitalar Lisboa Norte - Hospital de Santa Maria, tendo o período de recrutamento decorrido entre 30 de Julho e 14 de Agosto de 2014.

O único critério de inclusão utilizado foi o diagnóstico de LES de acordo com os critérios do Colégio Americano de Reumatologia de $1997 .{ }^{16}$ Como critérios de exclusão definiram-se: idade inferior a 18 anos, doença renal de outras etiologias que não nefrite lúpica, incluindo diabetes, e situações de turnover celular aumentado, nomeadamente, neoplasia activa e gravidez.

Este estudo foi aprovado pela Comissão de Ética do Centro Hospitalar Lisboa Norte, EPE, de acordo com a Declaração de Helsínquia.

\section{Variáveis avaliadas}

Para todos os doentes incluídos foram avaliados diversos parâmetros. A cistatina $C$ sérica foi determinada através da técnica de nefelometria (valores de referência disponibilizados pelo produtor: $0,5-0,96 \mathrm{mg} / \mathrm{dL}$ ) e a creatinina pelo método de Jaffe modificado (valores de referência disponibilizados pelo produtor: 0,7 - 1,3 mg/dL). A partir destes dois valores foi estimada a TFG para cada um dos doentes. A fórmula de estimativa da TFG considerada de referência foi a $C K D-E P I_{\text {creat, }}$, pois é actualmente a equação preconizada como preferida para calcular a TFG, segundo as recentes recomendações da KDIGO. ${ }^{2}$

Com o intuito de avaliar a sua influência nos níveis séricos de cistatina $C$, foram ainda registados os seguintes dados clínicos e laboratoriais: sexo, raça, idade, peso, altura, superfície corporal (calculada segundo a fórmula DuBois), ${ }^{17}$ hábitos tabágicos, função tiroideia (com base nos valores de hormona estimuladora da tiróide e tiroxina), proteína $\mathrm{C}$ reactiva $(P C R)$ e dose de corticoterapia que realizava no momento da colheita dos biomarcadores de função renal. Esta última foi convertida em todos os doentes em doses equivalentes de prednisolona.

\section{Análise estatística}

A totalidade da análise estatística foi efectuada utilizando o SPSS ${ }^{\circledR}$ (Statistical Package for the Social Sciences, versão 21).

A estatística descritiva das variáveis categóricas é apresentada como frequências e a das variáveis contínuas através da média e desvio padrão.

Foi utilizado um desenho emparelhado numa tabela 2 x 2 onde se classificou cada doente como tendo ou não elevação dos valores absolutos de cistatina $\mathrm{C}$ e/ou de creatinina (de acordo com os valores de referência do laboratório) e recorreu-se ao teste de McNemar para avaliar as semelhanças/diferenças encontradas, definindo-se $\alpha<0,05$ como estatisticamente significativo.

Foi realizada uma comparação gráfica entre cada uma das equações alternativas (TFGe) e a de referência (TFGr - CKD-EPI ${ }_{\text {creat }}$ ), através da plotagem entre a diferença nas equações (TFGe - TFGr) e a TFGe, representando-se ainda uma linha de regressão suavizada.

Para cada uma das equações alternativas, o viés foi definido como a diferença média (TFGe - TFGr), a precisão como o intervalo interquartil das diferenças (IQR) e a exactidão como a percentagem dos valores de TFGe que diferiam menos de $30 \%$ da TFGr (P30).

Dada a ausência de um método que possa ser definido como gold standard, não foi efectuada a análise de especificidade e sensibilidade de cada uma das equações alternativas, mas comparou-se a concordância entre cada uma das equações alternativas e a equação de referência, na classificação de DRC (TFGe $<60 \mathrm{~mL} / \mathrm{min} / 1,73 \mathrm{~m}^{2}$ ), através do teste de McNemar, definindo-se $\alpha<0,05$ como estatisticamente significativo.

Por fim, foram ainda analisados os diversos factores que poderiam influenciar a concentração sérica da cistatina $\mathrm{C}$ através de métodos de regressão linear, com análise univariada e ajuste multivariado, definindo-se $\alpha<0,05$ como estatisticamente significativo.

\section{RESULTADOS \\ Características da população}

Foram recrutados 37 doentes para participar no estudo, tendo sido todos incluídos por estarem de acordo com os critérios de inclusão e exclusão previamente estabelecidos.

As características da população estudada encontram-se discriminadas na Tabela 1. Os participantes eram predominantemente do sexo feminino $(94,6 \%)$ e de raça caucasiana $(94,6 \%)$, a média de idades verificada foi de 45,08 $( \pm 13,6)$ anos e a dose média de corticóides 8,31 ( $\pm 7,841)$ $\mathrm{mg}$. 
Tabela 1 - Características da população estudada. As variáveis contínuas encontram-se expressas pela média $(\mu) \pm$ desvio padrão $(\delta)$ e as variáveis categóricas através do número absoluto e percentagem.

\begin{tabular}{|c|c|c|c|}
\hline Característica & $\begin{array}{l}\text { Valor absoluto (\%) } \\
\qquad(n=37)\end{array}$ & $\mu \pm \delta$ & Variação \\
\hline Sexo feminino & $35(94,6 \%)$ & ------- & ------- \\
\hline \multicolumn{4}{|l|}{ Raça } \\
\hline Caucasiana & $35(94,6 \%)$ & -------- & ------- \\
\hline Negra & $2(5,4 \%)$ & -------- & -------- \\
\hline Hábitos tabágicos presentes & $6(16,2 \%)$ & ------- & ------- \\
\hline Alteração da função tiroideia & $2(5,4 \%)$ & ------- & ------- \\
\hline Idade (anos) & ------- & $45,08 \pm 13,63$ & $23-83$ \\
\hline Peso (Kg) & ------ & $69,22 \pm 16,39$ & $50-115$ \\
\hline Altura (m) & ------ & $1,65 \pm 0,07$ & $1,53-1,85$ \\
\hline Superfície corporal $\left(\mathrm{m}^{2}\right)$ & ------- & $1,73 \pm 0,20$ & $1,5-2,4$ \\
\hline Corticoterapia (mg/dia) & -------- & $8,31 \pm 7,84$ & $0-30$ \\
\hline PCR (mg/dL) & ------- & $0,44 \pm 0,60$ & $0,04-2,61$ \\
\hline
\end{tabular}

\section{Avaliação dos marcadores de função renal}

As frequências dos valores de creatinina e cistatina $C$ classificados como normais ou elevados para cada doente, tendo em conta dos valores de referência descritos, encontram-se registados na Tabela 2. Constatou-se uma elevação isolada da cistatina $\mathrm{C}$ em dez participantes, ao invés de nenhum caso de elevação isolada da creatinina. $\mathrm{O}$ teste McNemar demonstrou existir uma diferença estatisticamente significativa $(p=0,002)$ entre os dois métodos,

Tabela 2 - Frequência dos valores de creatinina e cistatina C classificados como normais ou elevados

\begin{tabular}{cccc}
\cline { 3 - 3 } & & \multicolumn{2}{c}{ Creatinina } \\
& Normal & Elevada \\
\hline \multirow{2}{*}{ Cistatina C } & Normal & 25 & 0 \\
& Elevada & 10 & 2 \\
\hline
\end{tabular}

favorecendo a elevação dos níveis de cistatina $\mathrm{C}$ em mais doentes.

Comparando as médias dos valores de TFGe calculados pelas diversas fórmulas com os do método de referência, constata-se que foram inferiores, à excepção da fórmula de Cockroft-Gault (Tabela 3).

Apesar de não ter sido efectuada a medição da TFG por um método gold standard, o viés, a exactidão e precisão (Tabelas 4 e 5) de cada equação foram calculados em

Tabela 3 - TFG estimada segundo as diferentes fórmulas

\begin{tabular}{lcc}
\hline Fórmula & Média $(\mu)$ & Desvio Padrão (ס) \\
\hline CKD- EPI $_{\text {Creat }}$ (TFGr) & 99,59 & 23,37 \\
CKD - EPI ${ }_{\text {Cist }}$ & 93,54 & 29,96 \\
CKD - EPICreat-cist & 97,19 & 27,54 \\
MDRD & 94,67 & 26,38 \\
Cockroft-Gault & 109,51 & 31,63 \\
\hline
\end{tabular}

Tabela 4 - Viés das diferentes fórmulas de estimativa da TFG

\begin{tabular}{lcccc}
\hline Fórmula & $\boldsymbol{\mu}$ & $\boldsymbol{\delta}$ & Intervalo de confiança 95\% & $\boldsymbol{p}$ - value \\
\hline CKD -EPI cist $_{\text {CKD }}$ EPI \\
creat-cist & $-6,0541$ & 19,7230 & {$[-12,6300,0,5219]$} & 0,070 \\
MDRD & $-2,4054$ & 12,1117 & {$[-6,4436,1,6328]$} & 0,235 \\
Cockroft-Gault & $-4,9216$ & 8,4265 & {$[-7,7311,-2,1121]$} & 0,001 \\
\hline
\end{tabular}

O viés foi calculado como a diferença média entre TFGe - TFGr $(\mu) ; \delta$ - desvio padrão 
Tabela 5 - Precisão e exactidão das diferentes fórmulas de estimativa da TFG

\begin{tabular}{lcc}
\hline Fórmula & IQR & P30 (\%) \\
\hline CKD -EPI ${ }_{\text {cist }}$ & 28,50 & 81,1 \\
CKD - EPI ${ }_{\text {creat-cist }}$ & 17,50 & 97,3 \\
MDRD & 9,50 & 100,0 \\
Cockroft-Gault & 32,85 & 81,1 \\
\hline
\end{tabular}

comparação com a TFGr. Verificou-se uma diferença estatisticamente significativa $(p<0,05)$ entre a TFGr e as TFGe com base nas fórmulas de Cockroft-Gault e MDRD, com um viés de $+9,9162(p=0,009)$ e $-4,9216(p<0,001)$, respectivamente, parecendo que a primeira sobrestima e a segunda subestima a TFG. Apesar da diferença não ser estatisticamente significativa, a equação CKD-EPI ${ }_{\text {cist }}$ apresenta também uma clara tendência para calcular TFGe inferiores à $\operatorname{TFGr}\left(-6,0541 \mathrm{~mL} / \mathrm{min} / 1,73 \mathrm{~m}^{2}, p=0,07\right)$. A MDRD foi a que melhor se correlacionou com o método padrão, sendo a mais exacta $(P 30=100)$ e a mais precisa $(I Q R=9,50)$.

Contudo, dado o CKD-EPI ${ }_{\text {creat }}$ não ser um verdadeiro gold standard, a determinação das medidas de viés (Tabela 4), precisão e exactidão (Tabela 5 ) não tem valor em
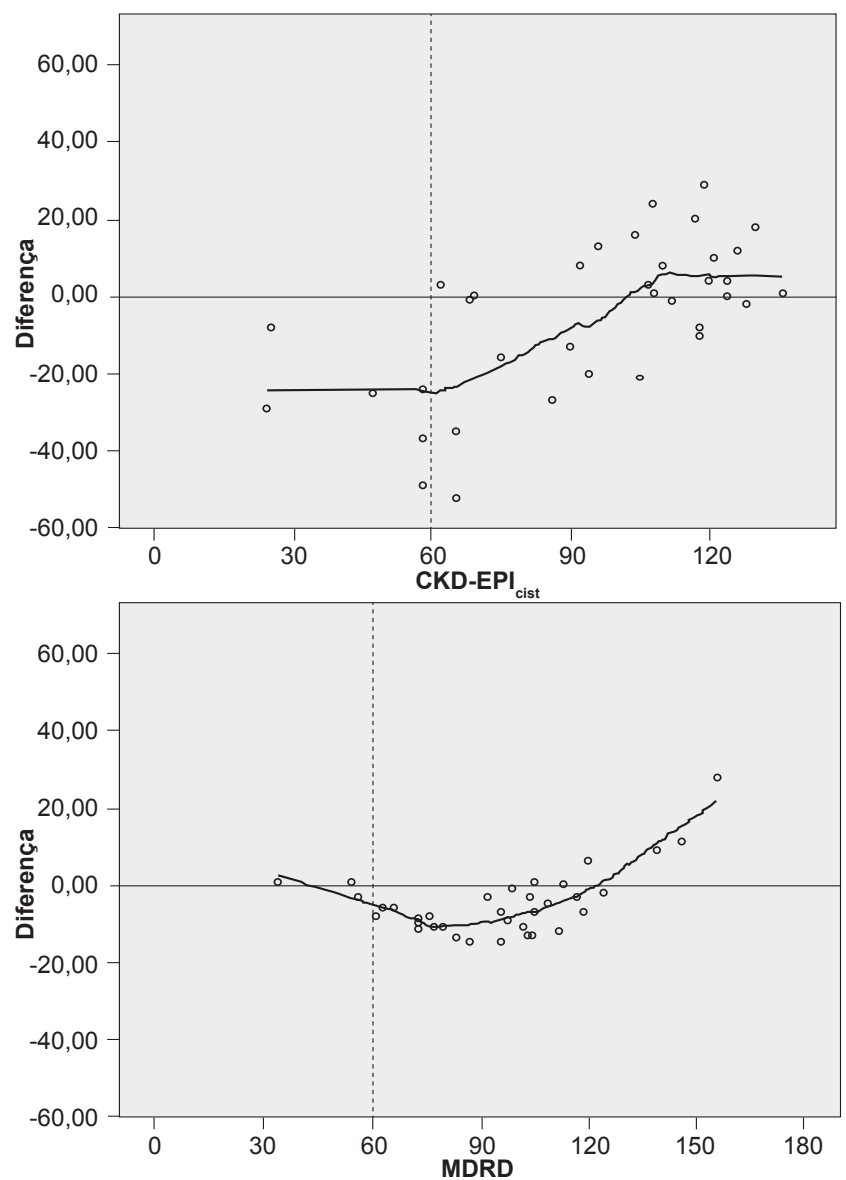

Figura 1 - Correlação entre a TFGe e a diferença (TFGr - TFGe) termos de definição do melhor método, mas permite-nos inferir se existe ou não diferença na determinação da função renal pelas várias equações.

A precisão foi calculada como o intervalo interquartil (IQR) e a exactidão foi calculada como a percentagem dos valores de TFGe que diferiam menos de $30 \%$ da $\mathrm{TFGr}$ (P30).

$\mathrm{Na}$ análise das diferenças entre a TFGr e a TFGe de cada uma das equações, ao longo do espectro de valores da TFGe (Fig. 1), destaca-se o facto das diferenças encontradas entre a TFGe pela CKD-EPI ${ }_{\text {cist }}$ e o método de referência serem mais evidentes para TFG mais baixas (perto do limiar dos $60 \mathrm{~mL} / \mathrm{min} / 1,73 \mathrm{~m}^{2}$ ), esbatendo-se para valores mais altos. As restantes fórmulas parecem manter uma diferença bastante mais uniforme e menos significativa ao longo do espectro de função renal. Como a dimensão da amostra era reduzida, não foi possível calcular Intervalos de Confiança a $95 \%$ ao longo de todo o espectro de TFGe, não podendo, por isso, concluir se a diferença é ou não estatisticamente significativa para cada valor de TFGe.

Comparando a classificação de DRC (definida neste estudo como TFG $<60 \mathrm{~mL} / \mathrm{min} / 1,73 \mathrm{~m}^{2}$ ) a partir das várias fórmulas com a obtida com o método de referência, verifica-se, apesar de não ser estatisticamente significativa ( $p=$ $0,375)$, efectivamente, uma diferença entre a classificação de DRC pela CKD-EPI ${ }_{\text {cist }}$ e pela CKD-EPI ${ }_{\text {creat }}$, tendo a equa-
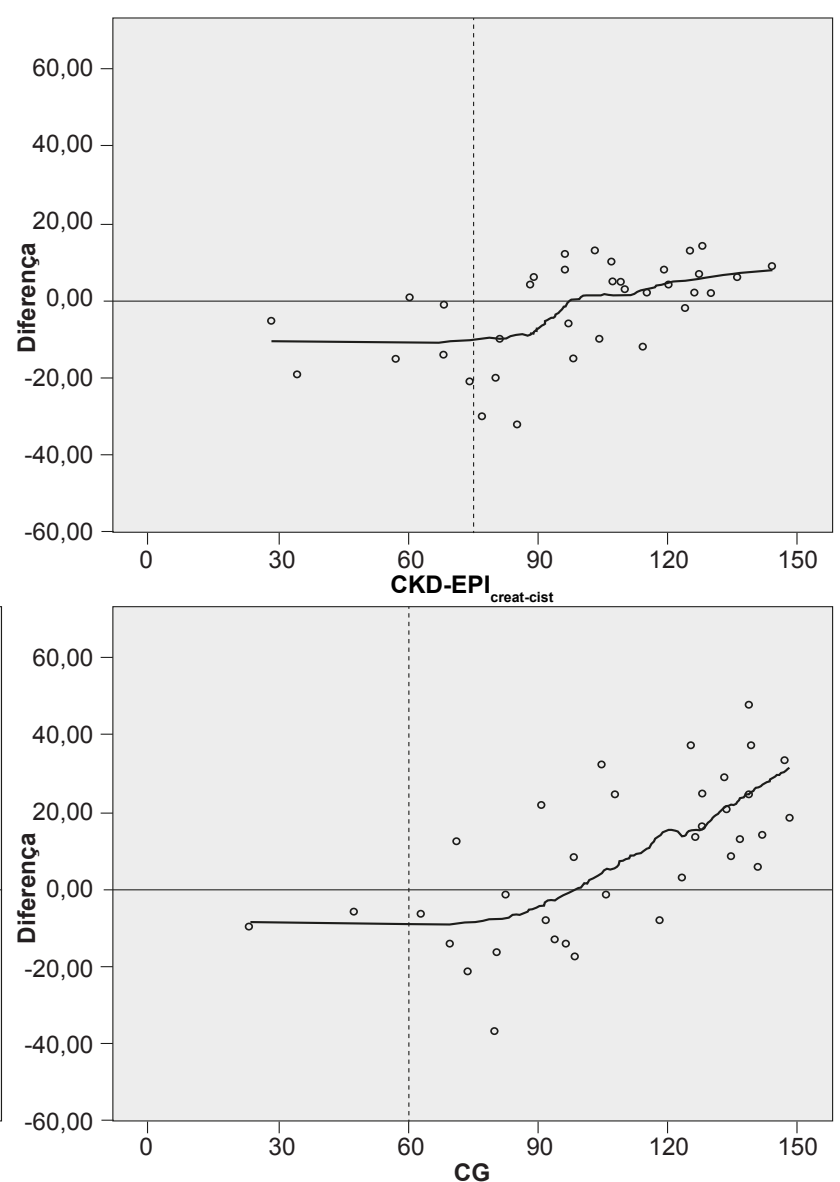
Tabela 6 - Presença ou ausência de DRC (TFG $\left.<60 \mathrm{~mL} / \mathrm{min} / 1,73 \mathrm{~m}^{2}\right)$ segundo as diferentes equações

\begin{tabular}{|c|c|c|c|c|c|}
\hline & & \multirow{2}{*}{\multicolumn{3}{|c|}{ CKD - EPI ${ }_{\text {creat }}$}} & \\
\hline & & & & & \multirow{2}{*}{$p$-value } \\
\hline & & TFG $<60$ & TFG $\geq 60$ & Total & \\
\hline \multirow{2}{*}{ CKD - EPI ${ }_{\text {cist }}$} & TFGe $<60$ & 2 & 4 & 6 & \multirow{2}{*}{$p=0,375$} \\
\hline & TFGe $\geq 60$ & 1 & 30 & 31 & \\
\hline \multirow{2}{*}{ CKD - EPI ${ }_{\text {creat-cist }}$} & $\mathrm{TFGe}<60$ & 3 & 1 & 4 & \multirow{2}{*}{$p=1$} \\
\hline & $\mathrm{TFGe} \geq 60$ & 0 & 33 & 33 & \\
\hline \multirow{2}{*}{ MDRD } & TFGe $<60$ & 3 & 0 & 3 & \multirow{2}{*}{$p=1$} \\
\hline & $\mathrm{TFGe} \geq 60$ & 0 & 34 & 34 & \\
\hline \multirow{2}{*}{ Cockroft-Gault } & TFGe $<60$ & 2 & 0 & 2 & \multirow{2}{*}{$p=1$} \\
\hline & $\mathrm{TFGe} \geq 60$ & 1 & 34 & 35 & \\
\hline
\end{tabular}

Tabela 7 - Análise de regressão univariada e multivariada

\begin{tabular}{lccccc}
\hline Característica & \multicolumn{2}{c}{ Univariada } & \multicolumn{2}{c}{ Multivariada } \\
& $\boldsymbol{r}$ & $\boldsymbol{p}$ - value & $\boldsymbol{\beta}$ & SE & $\boldsymbol{p}$ - value \\
\hline Idade (anos) & 0,745 & $<0,001$ & 0,019 & 0,004 & 0,001 \\
Superfície corporal $\left(\mathrm{m}^{2}\right)$ & 0,190 & 0,911 & $-0,171$ & 0,246 & 0,492 \\
Hábitos tabágicos & 0,104 & 0,541 & 0,031 & 0,130 & 0,813 \\
Corticoterapia (mg/dia) & 0,416 & 0,010 & 0,007 & 0,006 & 0,272 \\
PCR $(\mathrm{mg} / \mathrm{dL})$ & 0,126 & 0,457 & 0,056 & 0,76 & 0,464 \\
\hline
\end{tabular}

ção com base na cistatina $C$ reclassificado de novo quatro doentes como tendo DRC e um doente sido reclassificado como não tendo DRC. Em relação às outras fórmulas não se verifica qualquer diferença apreciável de classificação (Tabela 6).

\section{Outras variáveis que possam influenciar a cistatina C sérica}

$\mathrm{Na}$ avaliação de outros factores extra-renais que afectassem a concentração de cistatina $\mathrm{C}$ não foram analisados o sexo, a raça e a função tiroideia, dado o número ínfimo de doentes do sexo masculino, de raça negra ou com alterações da função tiroideia, o que impossibilitaria uma análise estatística credível.

A análise da regressão linear univariada (Tabela 7) mostra que os factores idade e corticoterapia se relacionam significativamente $(p<0,05)$ e positivamente com a cistatina $C$. Contudo, na análise de regressão com ajuste às múltiplas variáveis, das cinco variáveis analisadas, a única que influenciou a variação da cistatina $\mathrm{C}$ de forma estatisticamente significativa foi a idade.

\section{DISCUSSÃO}

No estudo CKD-EPI de 2012, comprovou-se que a equação que combina os dois marcadores (CKD-EPI creat-cist $_{\text {) }}$ ) constitui o método mais preciso e exacto para estimar a TFG, tendo reclassificado correctamente, acima ou abaixo do limite da TFG de $60 \mathrm{~mL} / \mathrm{min} / 1,73 \mathrm{~m}^{2}, 19 \%$ dos casos com TFG borderline $\left(45-74 \mathrm{~mL} / \mathrm{min} / 1,73 \mathrm{~m}^{2}\right)$ calculadas pela equação CKD-EPI creat , tendo este resultado sido estatisticamente significativo. ${ }^{7,18,19}$ Apesar disto, o viés foi semeIhante nas três equações.

Sendo assim, vários estudos demonstraram que a TFG $_{\text {cist }}$ melhora a definição de DRC, permitindo uma classificação e uma estratificação do risco mais exactas, comparativamente à $T F G_{\text {creat }}$. Com base nestas evidências, a KDIGO inclui nas suas mais recentes recomendações para a DRC (2012) várias sugestões relacionadas com a cistatina $\mathrm{C}$.

Nomeadamente, sugere-se a medição da cistatina $\mathrm{C}$ em adultos com $\mathrm{TFG}_{\text {creat }}$ entre $45-59 \mathrm{~mL} / \mathrm{min} / 1,73 \mathrm{~m}^{2}$ e que não possuam outros marcadores de lesão renal, quando necessária a confirmação de DRC. Sugere-se, ainda, a utilização da cistatina $C$ como teste confirmatório em situações clínicas específicas em que se preveja que a creatinina possa ser menos exacta ou possa ser influenciada por factores independentes da TFG.O facto destas sugestões ainda não constituírem recomendações prende-se com questões de aplicação prática, como o custo ou a menor disponibilidade. $^{2}$ 
Neste contexto, a cistatina $\mathrm{C}$ é apenas utilizada para aumentar a especificidade do diagnóstico de DRC. Contudo, a TFG ${ }_{\text {cist }}$ permite identificar casos de DRC não identificados pela TFG ${ }_{\text {creat' }}$, pelo que poderia ser utilizada para aumentar a sensibilidade do diagnóstico de DRC, como teste de rastreio. A sua viabilidade económica estaria dependente de factores como o custo do teste, a frequência de resultados positivos e a utilidade da detecção precoce de DRC, como por exemplo quais as implicações para o tratamento, bem como a probabilidade da creatinina sobrestimar a TFG. ${ }^{6}$ Assim, para que o rastreio com a cistatina $C$ pudesse ser vantajoso e custo-efectivo, a sua aplicação teria de ser específica, limitada, por exemplo, a populações com risco acrescido de DRC, sendo o caso mais investigado o da nefropatia diabética, tendo na maioria dos casos sido demonstrado superioridade deste novo marcador. ${ }^{20}$

Uma população a considerar seria a do lúpus eritematoso sistémico (LES). A lesão renal constitui um factor importante de morbilidade e mortalidade nestes doentes.

A fórmula que melhor se aplica a doentes com LES para estimar a função renal não foi ainda devidamente apurada. Dois estudos ${ }^{15,21}$ compararam as fórmulas de Cockroft-Gault e MDRD, com melhor desempenho desta última, tendo, no entanto, usado a CIC como gold standard e com uma elevada percentagem de colecções de urina de 24 horas inadequadas. Um outro estudo ${ }^{22}$ comparou diversas equações que utilizam a creatinina, tendo a CKD-EPI ${ }_{\text {creat }}$ como referência, tendo a MDRD obtido os melhores resultados. Contudo, sendo o LES uma doença com uma população muito específica, predominantemente do sexo feminino, com massa muscular reduzida, abrangendo diversas etnicidades, utilizadora de corticoterapia e outros fármacos imunossupressores específicos e com uma faixa de função renal muito variável, torna-se questionável se as fórmulas preconizadas para a população geral serão as que melhor se adaptam às particularidades destes doentes. ${ }^{13}$ A cistatina $C$, pelas evidências já referidas, poderá ser um marcador de função renal promissor nesta população em concreto, o que foi escassamente investigado na literatura médica, até à data. ${ }^{13,23}$

A principal preocupação na utilização deste marcador em doentes com LES prende-se com o facto de não existir ainda consenso quanto à influência que diversos factores relevantes nestes doentes possam ter na sua concentração sérica. Efectivamente, alguns estudos verificaram não existir uma correlação entre os valores de cistatina $\mathrm{C}$ e o uso de corticoesteróides ${ }^{23,24}$ ou o nível de actividade da doença. ${ }^{23}$ Contudo, Chew et $\mathrm{al}^{25}$ constataram que doentes com LES tinham níveis basais de cistatina $\mathrm{C}$ mais elevadosque o grupo controlo ( $p<0,0001)$, o que não se verificava com outros marcadores de função renal, e Lertnawapan et al ${ }^{26}$ apuraram uma correlação positiva e significativa entre a cistatina $\mathrm{C}$ e diversos marcadores de inflamação e de actividade da doença (PCR, VS, TNF-A, IL-6 e SLICC,). Resultados semelhantes foram observados em estudos com outras doenças reumatológicas, como a artrite reumatóide. ${ }^{26}$ É possível que esta associação reflicta o papel imunomodulador da cistatina $\mathrm{C}$, enquanto inibidor endógeno das cisteinoproteases, como as catepsinas ou as elastases, e da quimiotaxia de células polimorfonucleares, da libertação de radicais de oxigénio ou da fagocitose. Por outro lado, é difícil excluir a possibilidade de que a inflamação possa, por si só, levar a subtis declínios da função renal, eventualmente apenas detectados pela cistatina $\mathrm{C}$, já que esta é reconhecidamente mais exacta que a creatinina na identificação de ligeiras mudanças na TFG, o que reconheceria a cistatina $C$ como um melhor marcador de função renal em doentes com LES, nos quais o grau de envolvimento renal é extremamente variável.

Com base nestes dados, Martinez-Martinez ${ }^{13}$ procurou avaliar qual a melhor equação com base na creatinina, em doentes com LES. Numa primeira fase do seu estudo, comparou diversas equações (gold standard: clearance do iotalamato), tendo a equação com melhor desempenho sido a desenvolvida pelo Steven et al, ${ }^{27}$ que combinava a creatinina e a cistatina C. Contudo, a população analisada era constituída por apenas 14 participantes de nacionalidade mexicana, sendo estes doentes especificamente seleccionados pela baixa actividade da doença, pelas baixas doses de corticóides que faziam, por não terem hipotiroidismo e por serem não fumadores, de forma a garantir que estes factores não interferiam nos valores de cistatina $C$. Numa segunda fase do estudo, com 55 participantes, pretendeu-se determinar qual a melhor equação baseada apenas na creatinina, em comparação com a equação de Steven, ${ }^{27}$

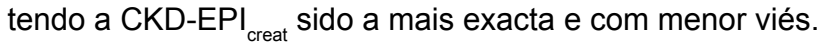

Os resultados obtidos na amostra deste estudo demonstram que existem diferenças entre a avaliação da função renal com base nos marcadores cistatina $\mathrm{C}$ e creatinina, em doentes com LES.

De facto, verificou-se uma diferença estatisticamente significativa entre o número de doentes nos quais os valores séricos de cistatina $C$ e de creatinina se encontravam elevados, favorecendo mais vezes a elevação isolada dos níveis de cistatina $C(p=0,002)$. Concordantemente, apesar da diferença não ser estatisticamente significativa, a equação CKD-EPI ${ }_{\text {cist }}$ apresenta uma clara tendência para estimar TFG inferiores à CKD-EPI ${ }_{\text {creat }}\left(-6,0541 \mathrm{~mL} / \mathrm{min} / 1,73 \mathrm{~m}^{2}\right.$ $p=0,07)$.

Dada a ausência de um verdadeiro método gold standard de medição da TFG, não podemos inferir qual dos marcadores se encontra mais próximo da realidade, apenas constatamos que nos permitem obter resultados diferentes. Contudo, é vastamente conhecido que a creatinina estima valores de TFG relativamente imprecisos, devido aos seus determinantes independentes da filtração glomerular. Este facto pode ser particularmente relevante nos doentes com LES, que apresentam tendencionalmente pouca massa muscular, quer pela forte predominância do sexo feminino, quer pelo processo de doença crónica em si e pelo uso prolongado de corticoterapia, o que conduz a uma menor produção de creatinina, podendo justificar uma possível sobrestimação da TFG pelas fórmulas derivadas da creatinina 
Destaca-se ainda o facto da diferença entre os dois métodos ser mais evidente para TFG mais baixas (perto do limiar de $60 \mathrm{~mL} / \mathrm{min} / 1,73 \mathrm{~m}^{2}$ ), dado com relevância clínica, já que a TFG ${ }_{\text {cist }}$ permitiu reclassificar de novo quatro doentes como tendo DRC e um doente como não tendo DRC. Contudo, esta diferença não apresentou significância estatística $(p=0,375)$. Estes resultados estão de acordo com as evidências literárias que apontam a cistatina $\mathrm{C}$ como marcador mais sensível de discretas alterações da TFG, em relação à creatinina, ${ }^{15,25}$ detectando mais precocemente a DRC e podendo, portanto, ser um melhor método de rastreio, especialmente em populações cuja TFG estimada a partir da creatinina possa não ser tão precisa, ${ }^{2}$ como parece ser o caso de doentes com LES.

Por outro lado, a correlação entre determinados factores, presentes na população com LES, como o uso de corticoterapia ou a inflamação, e a variação dos níveis séricos de cistatina $C$, não está completamente estabelecida. Neste estudo, os factores idade e corticoterapia relacionaram-se significativamente $(p<0,05)$ e positivamente com a cistatina $C$ na análise univariada, tendo, no entanto, na análise com ajuste multivariável, apenas a idade demonstrado uma correlação estatisticamente significativa. Contudo, existindo um declínio fisiológico da TFG com o processo de enveIhecimento, esta parece tratar-se de um factor confundente, pelo que, neste estudo, a cistatina $C$ não parece ter sido influenciada significativamente por nenhum factor, além da função renal. No entanto, as doses de corticóides utilizadas pelos participantes neste estudo eram relativamente baixas $(8,31 \pm 7,8410$, variando entre [0 - 30] $\mathrm{mg})$ e dada a pequena dimensão da amostra, mais estudos de correlação são necessários para se excluir definitivamente a influência que estes e outros factores possam ter nos níveis de cistatina $\mathrm{C}$, conduzindo a uma eventual subestimativa dos valores de TFG $_{\text {cist }}$.

\section{Pontos fortes e limitações do estudo}

A limitação principal deste estudo prende-se com a ausência de um método gold standard de medição de TFG com o qual comparar as restantes equações avaliadas, pela sua complexidade de execução técnica e financeira. Este problema é minorado pelo facto da equação CKD-EPI ${ }_{\text {creat, }}$ utilizada como referência, ter demonstrado uma elevada concordância com a TFG medida através de marcadores de filtração exógenos. ${ }^{8}$ Serão necessários estudos que utilizem um gold standard de medição da função renal, para comprovar a validade dos resultados preliminares, obtidos neste estudo.

Por outro lado, a população estudada é reduzida e apresenta pouca diversidade étnica ( $94,6 \%$ caucasianos) e de género (94,6\% sexo feminino). Contudo, estes factores estão de acordo com as particularidades epidemiológicas do LES, no qual a prevalência da doença entre o sexo feminino e o masculino é de 9:1, e da população no nosso país, naturalmente de predomínio caucasiano.
Além disso, comparativamente ao estudo semelhante, realizado por Martinez-Martinez, ${ }^{13}$ este estudo apresenta a vantagem de possuir um número superior de participantes e uma população muito menos seleccionada (em comparação à fase 1). De facto, dos 37 doentes avaliados inicialmente, todos foram incluídos no estudo, de acordo com os critérios de inclusão e exclusão estabelecidos, pelo que este estudo poderá ter uma boa validade externa, representativa da prática clínica quotidiana, podendo aplicar-se à generalidade da população dos doentes com LES em Portugal.

\section{CONCLUSÃO}

É importante continuar a investigar métodos mais fiáveis de determinação de função renal e de estratificação do risco, especialmente em populações susceptíveis, como o caso do LES. Os resultados deste estudo apoiam a hipótese de que a cistatina $C$ sérica é um marcador de lesão renal diferente da creatinina sérica, podendo ser um marcador mais sensível de discretas alterações da TFG e potencialmente superior à creatinina como método de rastreio de DRC, quer pela precocidade do diagnóstico, quer pelo seu maior valor prognóstico. Este facto tem implicações clínicas importantes particularmente nos doentes com LES, cujas complicações renais são um dos principais factores de morbilidade e mortalidade. Contudo, por um lado, com base neste estudo, não pode ser inferido que a cistatina $C$ sérica é superior à creatinina sérica, na medida em que a TFG não foi avaliada através da clearance de um marcador de filtração exógeno (gold standard). Por outro lado, a cistatina $\mathrm{C}$ é um marcador dispendioso comparativamente com a creatinina e não amplamente disponível na prática clínica, o que pode ser limitativo da sua aplicabilidade na prática clínica em Portugal.

\section{PROTECÇÃO DE PESSOAS E ANIMAIS}

Os autores declaram que os procedimentos seguidos estavam de acordo com os regulamentos estabelecidos pelos responsáveis da Comissão de Investigação Clínica e Ética e de acordo com a Declaração de Helsínquia da Associação Médica Mundial.

\section{CONFIDENCIALIDADE DOS DADOS}

Os autores declaram ter seguido os protocolos do seu centro de trabalho acerca da publicação dos dados de doentes.

\section{CONFLITO DE INTERESSES}

Os autores declaram não ter nenhum conflito de interesses relativamente ao presente artigo.

\section{FONTES DE FINANCIAMENTO}

Não existiram fontes externas de financiamento para a realização deste artigo. 


\section{REFERÊNCIAS}

1. Sarnak M, Levey A, Schoolwerth A, Coresh J, Culleton B, Hamm LL, et al. Kidney disease as a risk factor for development of cardiovascular disease. Circulation. 2003;108:2154-69.

2. Kidney Disease: Improving Global Outcomes (KDIGO) CKD Work Group. KDIGO 2012 Clinical Practice Guideline for the Evaluation and Management of Chronic Kidney Disease. Kidney Int. 2013;3:1-150.

3. McMahon GM, Waikar SS. Biomarkers in nephrology: core curriculum. Am J Kidney Dis. 2013;62:165-78.

4. Herget-Rosenthal S, Bokenkamp A, Hofmann W. How to estimate GFR - serum creatinine, serum cystatin $C$ or equations? Clin Biochem. 2007;40:153-61.

5. Rahn $\mathrm{KH}$, Heidenreich $\mathrm{S}$, Brükner D. How to assess glomerular function and damage in humans. J Hypertens. 1999;17:309-17.

6. Shlipak MG, Mattes MD, Peralta CA. Update on cystatin C: incorporation into clinical practice. Am J Kidney Dis. 2013;62:595 -603.

7. Tidman $M$, Sjöström P, Jones I. A comparison of GFR estimating formulae based upon s-cystatin $\mathrm{C}$ and s-creatinine and a combination of the two. Nephrol Dial Transplant. 2008;23:154-60.

8. Levey AS, Stevens LA, Schmid CH, Zhang YL, Castro AF 3rd, Feldman $\mathrm{HI}$, et al. A new equation to estimate glomerular filtration rate. Ann Intern Med. 2009;150:604-12.

9. Chew J, Saleem M, Florkowski CM, George PM. Cystatin C - a paradigm of evidence based laboratory medicine. Clin Biochem Rev. 2008;29:4762.

10. Dharnidharka VR, Kwon C, Stevens G. Serum cystatin C is superior to serum creatinine as a marker of kidney function: a meta-analysis. Am J Kidney Dis. 2002;40:221-6.

11. Lertnawapan $R$, Bian A, Rho $Y H$, Raggi $P$, Oeser A, Solus JF, et al. Cystatin $C$ is associated with inflammation but not atherosclerosis in Systemic Lupus Erithematosus. Lupus. 2011;21:279-87.

12. Rajashekar A, Perazella MA, Crowley S. Systemic diseases with renal manifestations. Prim Care. 2008;35:297-328.

13. Martínez-Martínez MU, Mandeville P, Llamazares-Azuara L, AbudMendoza C. CKD-EPI is the most reliable equation to estimate renal function in patients with systemic lupus erythematosus. Nefrologia. 2013;33:99-106.

14. Gordon C, Jayne D, Pusey C. European consensus statement on the terminology used in the management of lupus glomerulonephritis. Lupus. 2009;18:257-63.

15. Kasitanon N, Fine DM, Haas M, Magder LS, Petri M. Estimating renal function in lupus nephritis: comparison of the modification of diet in renal disease and Cockcroft Gault equations. Lupus. 2007;16:887-95.

16. Hochberg MC. Updating the American College of Rheumatology revised criteria for the classification of systemic lupus erythematosus. Arthritis Rheum. 1997;40:1725.

17. DuBois D, DuBois EF. A formula to estimate the approximate surface area if height and weight be known. Arch Intern Med. 1916;17:863-71.

18. Inker LA, Schmid CH, Tighiouart H, Eckfeldt JH, Feldman HI, Greene $\mathrm{T}$, et al. Estimating glomerular filtration rate from serum creatinine and cystatin C. N Engl J Med. 2012;367:20-9.

19. Peralta CA, Shlipak MG, Judd S, Cushman M, McClellan W, Zakai NA, et al. Detection of chronic kidney disease with creatinine, cystatin C, and urine albumin-to-creatinine ratio and association with progression to end-stage renal disease and mortality. JAMA. 2011;305:1545 -52.

20. Perkins BA, Nelson RG, Ostrander BE, Blouch KL, Krolewski AS, Myers $B D$, et al. Detection of renal function decline in patients with diabetes and normal or elevated GFR by serial measurements of serum cystatin C concentration: results of a 4-year follow-up study. J Am Soc Nephrol. 2005;16:1404-12.

21. Leung YY, Lo KM, Tam LS, Szeto CC, Li EK, Kun EW. Estimation of glomerular filtration rate in patients with systemic lupus erythematosus. Lupus. 2006;15:276-81.

22. Martínez-Martínez MU, Borjas-García JA, Magaña-Aquino M, CuevasOrta E, Llamazares-Azuara L, Abud-Mendoza C. Renal function assessment in patients with systemic lupus erythematosus. Rheumatol Int. 2012;32:2293-9.

23. Martínez-Martínez MU, Abud-Mendoza C. Cistatina C sérica como marcador de función renal en pacientes com lupus eritematoso sistémico. Reumatol Clin. 2014;8:158-61.

24. Silva MV, Moscoso-Solorzano G, Nishida SK, Kirsztain GM. Os níveis séricos de cistatina $C$ sofrem influência da dose de corticóide em pacientes com nefrite lúpica? J Bras Nefrol. 2011;33:306-12.

25. Chew C, Permberton P, Husain A, Haque S, Bruce IN. Serum cystatin C is independenty associated with renal impairment and high sensitivity $C$ - reactive protein in systemic lupus erythematosus. Clin Exp Rheumatol. 2013;31:251-5.

26. Lertnawapan R, Bian A, Rho YH, Kawai VK, Raggi P, Oeser A, et al. Cystatin $\mathrm{C}$, renal function and atherosclerosis in rheumatoid arthritis. J Rheumatol. 2011;38:2297-300.

27. Stevens LA, Coresh J, Greene T, Levey AS. Assessing kidney functionmeasured and estimated glomerular filtration rate. $\mathrm{N}$ Engl $\mathrm{J}$ Med. 2006;354:2473-83 
Lígia PEIXOTO, Patrício AGUIAR, Raquel de BRAGANÇA, Joana Rosa MARTINS, Alba Janeiro ACABADO, José Luís DUCLA-SOARES

\section{Cistatina C: Um Marcador de Função Renal Promissor em Doentes com Lúpus Eritematoso Sistémico?}

Acta Med Port 2015:28:333-341

Publicado pela Acta Médica Portuguesa, a Revista Científica da Ordem dos Médicos

Av. Almirante Gago Coutinho, 151

1749-084 Lisboa, Portugal.

Tel: +351218428 215

E-mail: submissao@actamedicaportuguesa.com

www.actamedicaportuguesa.com

ISSN:0870-399X | e-ISSN: 1646-0758

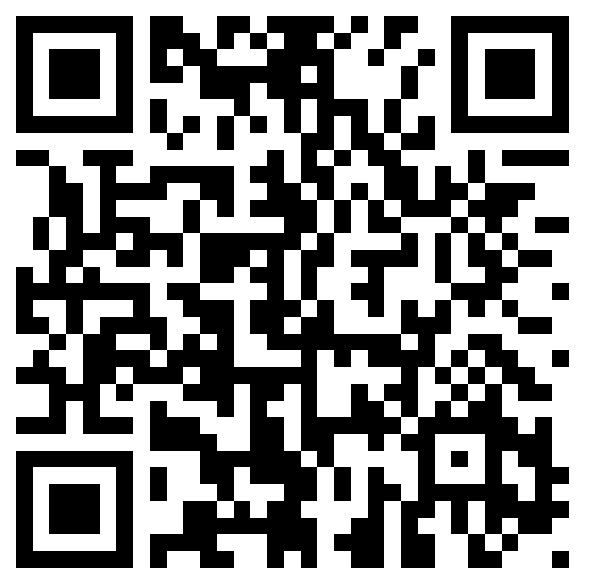

ACTA MÉDICA

PORTUGUESA 